Psychotherapeut 2021 $66: 119-126$ https://doi.org/10.1007/s00278-020-00489-9 Angenommen: 14. Dezember 2020 Online publiziert: 17. Januar 2021

๑) Der/die Autor(en) 2021
Elisa Wegmann ${ }^{1} \cdot$ Kim-Sarah Jung $^{1} \cdot$ Stephanie Antons ${ }^{1,2}$

${ }^{1}$ Allgemeine Psychologie: Kognition und Center for Behavioral Addiction Research (CeBAR), Universität Duisburg-Essen, Duisburg, Deutschland

${ }^{2}$ Erwin L. Hahn Institute for Magnetic Resonance Imaging, Essen, Deutschland

\title{
Problematische Nutzung sozialer Netzwerke
}

\author{
Bedeutung suchtrelevanter Konstrukte wie \\ Impulsivität, Erleben von Craving und \\ Inhibitionskontrolle
}

\begin{abstract}
Die Nutzung sozialer Netzwerke wie Facebook, WhatsApp und Instagram ist ein wesentlicher Bestandteil des Alltags. Sie ermöglicht den synchronen und asynchronen, kommunikativen Austausch von Inhalten, Bildern und Videos. Die Nutzung erfolgt zumeist zielgerichtet und funktional, wobei Vorteile und positive Folgen erfahren werden. Zunehmend wird jedoch auch von subjektiven Beeinträchtigungen und negativen Konsequenzen aufgrund einer exzessiven, unkontrollierten Nutzung berichtet, die Parallelen zu anderen internetbezogenen Verhaltenssüchten und substanzgebundenen Störungen aufweisen.
\end{abstract}

\section{Einleitung}

Die Nutzung sozialer Netzwerke ist vielfältig und kann bedürfnisorientiert erfolgen, denn sie bietet die Möglichkeit, mit fremden und auch bekannten Personen zu kommunizieren, Interessen zu verfolgen, zwischenmenschliche Beziehungen aufrechtzuerhalten, aber auch Informationen preiszugeben (Boyd und Ellison 2007). Doch neben den positiven Aspekten gibt es zunehmend auch negative Formen der Nutzung sozialer Netwerke, die auf einen dysfunktionalen Gebrauch hinweisen (z. B. Hatespeech, Cyberbullying). Zusätzlich wird die suchtartige, unkontrollierte Nutzung des Smartphones generell, aber auch sozialer Netzwerke im Spezifischen, als mögliche Verhaltenssucht diskutiert (Montag et al. 2019). Dabei orientiert sich die Beschreibung der problematischen, suchtartigen Nutzung von sozialen Netzwerken an der Definition des pathologischen Glücksspielens und Spielens von Videospielen und umfasst Symptome wie die Nutzung der Anwendung als Hauptbeschäftigung, Toleranzentwicklung, Emotionsregulation (z. B. Erleben positiver Gefühle oder Vermeidung negativer Emotionen), Entzugssymptome, Inkaufnahme negativer Konsequenzen und Konflikte sowie fehlgeschlagene Versuche, die Nutzung einzuschränken (Andreassen 2015; Kuss und Griffiths 2011). Gleichzeitig muss bei der Betrachtung des Phänomens berücksichtigt werden, dass, im Gegensatzzu pathologischem Glücksspielen und Spielen von Videospielen, die problematische Nutzung sozialer Netzwerke noch keinen Einzug in die gängigen Klassifikationssysteme wie die 5. Auflage des Diagnostic and Statistical Manual of Mental Disorders (DSM-5; American Psychiatric Association 2013) und die 11. Ausgabe der Internationalen statistischen Klassifikation der Krankheiten und verwandter Gesundheitsprobleme (ICD11; World Health Organization 2020) erhielt. Zusätzlich fehlt es an einer einheitlichen Definition in der Forschung, einem Diagnosestandard und einer einheitlichen Bezeichnung des Störungsbildes.
Um jedoch die klinische und gesellschaftliche Bedeutsamkeit des Phänomens einer problematischen, suchtartigen Nutzung sozialer Netzwerke genauer zu verstehen und einzuordnen, erscheint es von äußerster Relevanz, die Entstehungs- und Aufrechterhaltungsmechanismen dieses spezifischen Störungsbildes zu identifizieren. In ihrem Übersichtsbeitrag formulieren Brand et al. (2020) 3 Kriterien als Orientierungshilfe, worauf bei der Deklaration und dem Verständnis eines möglichen suchtartigen Verhaltens $\mathrm{zu}$ achten ist. Diese umfassen 1) die Ermittlung der klinischen Relevanz, 2) die theoretische Einordnung und 3) das Vorhandensein empirischer Evidenz hinsichtlich zugrunde liegender Mechanismen.

In Bezug auf den ersten Punkt illustrieren verschiedene Umfragen, dass Menschen von einer subjektiven Beeinträchtigung im Alltag aufgrund der unkontrollierten Nutzung sozialer Netzwerke und dem damit verbundenen Erleben negativer Konsequenzen berichten. Laut einer Studie der DAKGesundheit (2018) schildern Kinder und Jugendliche zunehmend Schlafstörungen, Konflikte mit den Eltern sowie den Verlust anderer Interessen. Weltweite Schätzungen berichten von Prävalenzen einer problematischen Nutzung zwischen 8,6 und $41,9 \%$, wobei die stark variierenden Zahlen auf die fehlende diagnostische Sicherheit zurückzuführen sind (Guedes et al. 2016). Darüber hinaus 
unterstreichen Studien die Bedeutsamkeit psychopathologischer Symptome und zeigen den Zusammenhang zwischen der problematischen Nutzung sozialer Netzwerke und Depressivität, Ängstlichkeit, Aufmerksamkeitsdefizitund Hyperaktivitätsstörung sowie parallelem Substanzmissbrauch (Clayton et al. 2013; Moreau et al. 2015). Weitere prädisponierende Faktoren sind soziale Defizite, Einsamkeitsempfinden, Minderwertigkeitsgefühle, ein geringeres Selbstwertgefühl und auch eine erhöhte Stressvulnerabilität (Wegmann und Brand 2019).

Betrachtet man den zweiten Punkt der theoretischen Einordnung eines Störungsbildes, wurden bereits verschiedene theoretische Ansätze aus der Suchtforschung, wie dem Dual-Prozess-Modell von Bechara (2005), der „Incentive Sensitization Theory" (Robinson und Berridge 2008) und damit assoziierte Konstrukte wie Reizreaktivität („cue reactivity“) und Craving (Carter und Tiffany 1999) genutzt, um zugrunde liegende Mechanismen verschiedener Verhaltenssüchte oder auch spezifischer Störungsbilder zu erklären (Beitrag von Brand in diesem Heft). Auch zur Erforschung der problematischen Nutzung sozialer Netzwerke wurden diese bereits als Grundlage herangezogen und erweitert (Wegmann und Brand 2019). Zentrale Konstrukte, die immer wieder hervorgehoben werden, lassen sich den kognitiven und affektiven Prozessen zuordnen. Diese umfassen eine erhöhte Sensitivität gegenüber suchtrelevanten Reizen (z. B. Aufmerksamkeitsverzerrungen und Reizreaktivität) und dem damit einhergehenden Erleben von Craving, implizite Kognitionen sowie die Beeinträchtigung kognitiver Funktionen wie Inhibitionskontrolle und weiterer Exekutivfunktionen. Empirische Arbeiten unterstreichen die Bedeutsamkeit von Reizreaktivität und Craving für verschiedene Verhaltenssüchte (Starcke et al. 2018). Auch für die problematische Nutzung sozialer Netzwerke konnte bereits ein Zusammenhang zwischen der Symptomschwere und dem Erleben von Craving nach der Konfrontation mit spezifischen, auditiven und visuellen Reize gezeigt werden (Wegmann et al. 2018). Die Unterdrückung automa- tischer Reaktionen auf einen Reiz bzw. die Schwierigkeit der Unterdrückung ist ebenfalls eine wichtige kognitive Komponente. Dabei wird zwischen einer generellen und einer spezifischen Inhibitionskontrolle, also Inhibition einer automatisierten Handlung bei der Konfrontation mit einem suchtspezifischen Reiz, differenziert. Empirische Arbeiten illustrierten die Bedeutsamkeit von Impulsivität und der spezifischen Inhibitionskontrolle für verschiedene Störungsbilder wie suchtartiges Spielen von Videospielen und problematische Nutzung von Online-Pornografie, wobei verschiedene experimentelle Paradigmen wie das GoNoGo-Paradigma und die Stopp-SignalAufgabe genutzt wurden (z. B. Antons und Brand 2018; Kim et al. 2017). Antons und Brand (2018) betonen die Bedeutsamkeit der (interagierenden) Betrachtung von Impulsivität, dem Erleben von Craving und der spezifischen Inhibitionskontrolle bei der problematischen Pornografienutzung. Auch im Kontext einer problematischen Nutzung sozialer Netzwerke wurde bereits die Relevanz von Impulsivität und spezifischer Inhibitionskontrolle (gemessen mit modifizierten Versionen des Go-NoGo-Paradigmas) gezeigt (Gao et al. 2019; Wegmann et al. 2020). Betrachtet man diese ersten empirischen Ergebnisse unter Berücksichtigung der empirischen Evidenzlage weiterer kognitiver Komponenten für das potenzielle Störungsbild wird deutlich, dass zentrale Konstrukte wie Impulsivität, Reizreaktivität und Craving als auch Inhibitionskontrolle relevante Komponenten bei einer problematischen Nutzung sozialer Netzwerke darstellen (Wegmann und Brand 2020). Für die Einordnung des Störungsbildes als mögliche suchtartige Verhaltensweise betonen die Autoren jedoch auch, dass weitere empirische Studien unter Berücksichtigung theoretischer Modelle und einer methodischen Vielfalt notwendig sind.

\section{Ziel der Arbeit}

Die Relevanz affektiver und kognitiver Komponenten wie Impulsivität, Reizreaktivität und Craving sowie spezifischer Inhibitionskontrolle soll mithilfe einer modifizierten Version der Stopp-Signal-
Aufgabe untersucht werden. Zusätzlich werden Interaktionseffekte geprüft. Der Einsatz der Stopp-Signal-Aufgabe als maßgebliche Neuerung soll die Inhibitionskontrolle bei gleichzeitiger Präsentation von anwendungsspezifischen Bildreizen adressieren. Innerhalb der Aufgabe wird eine bereits initiierte motorische Handlung inhibiert. Im Vergleich zur Go-NoGo-Aufgabe setzt die Stopp-Signal-Aufgabe später im Handlungsprozess an (Bari und Robbins 2013). Für die Handlungskontrolle sind die parallel gezeigten Bildreize nicht relevant, jedoch wird eine Interaktion zwischen den Prozessen der Handlungskontrolle und denen der Verarbeitung der Bilder, die Bildausschnitte von OnlineKommunikationsanwendungen zeigen, vermutet. Dabei können nicht nur visuelle, sondern auch auditive Reize zur Reizreaktivität und zum Erleben von Craving führen (Carter und Tiffany 1999). So kann sowohl das Hören eines Klingeltons als auch die Nachricht auf dem Bildschirm bewirken, dass Personen mit einer problematischen Nutzung mit einem stärkeren Verlangen reagieren, diese Online-Anwendung nun auch zu nutzen. Somit wurden im Vorfeld folgende Hypothesen angenommen:

1. Es gibt einen signifikanten Unterschied zwischen dem subjektiv erlebten Craving vor und nach dem Cue-reactivity-Paradigma sowie nach der Stopp-Signal-Aufgabe.

2. Es gibt einen signifikanten Zusammenhang zwischen der Impulsivität, dem Erleben von Craving sowie der spezifischen Inhibitionskontrolle und der Tendenz einer problematischen Nutzung sozialer Netzwerke.

3. Der Effekt von Impulsivität auf die Tendenz einer problematischen Nutzung sozialer Netzwerke wird durch affektive und kognitive Komponenten wie das erlebte Craving nach dem Cue-reactivity-Paradigma und die spezifische Inhibitionskontrolle moderiert. 
Psychotherapeut 2021 -66:119-126 https://doi.org/10.1007/s00278-020-00489-9

(c) Der/die Autor(en) 2021

\section{E. Wegmann $\cdot$ K.-S. Jung $\cdot$ S. Antons}

\section{Problematische Nutzung sozialer Netzwerke. Bedeutung suchtrelevanter Konstrukte wie Impulsivität, Erleben von Craving und Inhibitionskontrolle}

\section{Zusammenfassung}

Hintergrund. Die Nutzung sozialer Netzwerke ist ein zentraler Bestandteil des Alltags. Neben vielen Vorteilen wird jedoch zunehmend von subjektiven Beeinträchtigungen und negativen Konsequenzen aufgrund einer exzessiven, unkontrollierten Nutzung berichtet. Parallelen zu anderen internetbezogenen Verhaltenssüchten und substanzgebundenen Störungen lassen vermuten, dass Konstrukte wie Impulsivität, das Erleben von Craving und eine beeinträchtige Inhibitionskontrolle ebenfalls Risikofaktoren bei der Entwicklung und Aufrechterhaltung einer problematischen Nutzung sozialer Netzwerke darstellen. Material und Methode. In der vorliegenden Experimentalstudie mit 64 Teilnehmenden im Alter von 18 bis 59 Jahren wurden ein auditives "Cue-reactivity"-Paradigma sowie eine modifizierte Version der Stopp-SignalAufgabe zur Erfassung von Reizreaktivität und Inhibitionskontrolle eingesetzt. Zur Erhebung von Craving, Impulsivität und der Symptomschwere einer problematischen Nutzung sozialer Netzwerke wurden Fragebogen verwendet.

Ergebnisse. Die Ergebnisse zeigen, dass die Tendenz einer problematischen Nutzung mit einer höheren Impulsivität und Craving einhergeht. Die Inhibitionskontrolle sowie die Interaktion zwischen den verschiedenen Konstrukten konnten keine weitere Beiträge zur Varianzaufklärung der Symptomschwere leisten.
Schlussfolgerung. Die Ergebnisse illustrieren die Bedeutsamkeit von Impulsivität und Craving bei der Entwicklung und Aufrechterhaltung einer problematischen Nutzung sozialer Netzwerke. Darüber hinaus gilt es, die Spezifität der einzelnen Reize hinsichtlich des möglichen Konfliktpotenzials und Aufforderungscharakters sowie die damit einhergehende Relevanz spezifischer kognitiver Komponenten in weiteren Studien zu prüfen.

Schlüsselwörter

Verhaltenssucht · Smartphonesucht . Impulsivität· Inhibitionskontrolle · Internet

\section{Problematic use of social networks. Importance of addiction-relevant constructs, such as impulsivity, experience of craving and inhibitiory control}

\section{Abstract}

Background. The usage of social networks is a central part of daily life. In addition to many advantages, subjective impairments and negative consequences due to an excessive, uncontrolled use are increasingly being reported. Parallels to other internetrelated addictive behavior and substance use disorders suggest that constructs, such as impulsiveness, the experience of craving and impairments in inhibitory control are risk factors for the development and maintenance of a problematic social network use.

Material and methods. In this experimental study consisting of 64 participants aged be- tween $18-59$ years, an auditory cue-reactivity paradigm and a modified version of the stopsignal task were used to address cue reactivity and inhibitory control. Questionnaires were used to measure craving and impulsiveness as well as the symptom severity of problematic social network use.

Results. The results show that the tendency towards a problematic use is related to greater impulsiveness and craving experiences. The inhibitory control as well as the interaction between the different constructs could not contribute further to variance explanation of symptom severity.
Conclusion. The results illustrate the importance of impulsiveness and the experience of craving in developing and maintaining a problematic use of social networks. In addition, the specificity of certain stimuli with respect to the possible conflict and responding character and the associated relevance of specific cognitive components should be examined in further studies.

Keywords

Addictive behavior - Smartphone addiction . Impulsivity · Inhibitiory control · Internet

\section{Material und Methoden}

\section{Stichprobe}

Die Stichprobe bestand aus 64 Teilnehmenden (41 weiblich) im Alter von 18 bis 59 Jahren (Mittelwert $[\mathrm{M}] \pm$ Standardabweichung $[S D]=25,7 \pm 10,4$ Jahre), von denen $9,6 \%$ über mindestens einen Fachhochschulabschluss, 78,1 \% über die allgemeine Hochschulreife und 12,3\% über einen Universitätsabschluss verfügten. Die Stichprobe umfasste $\mathrm{zu}$ $76,6 \%$ Studierende, während die restli- chen 23,4\% angaben, Arbeitnehmende oder selbstständig zu sein. Alle Teilnehmenden nutzten soziale Netzwerke wie WhatsApp, Instagram, Twitter, Facebook, iMessage, Threema oder Snapchat. Die selbsteingeschätze Nutzungszeiten pro Tag betrugen zwischen 17 und 630 min. Die durchschnittliche Nutzung betrug $181,5 \pm 142,7 \mathrm{~min}$.

\section{Untersuchungsablauf}

Die Rekrutierung der Stichprobe erfolgte an der Universität Duisburg-Essen und im privaten Umfeld der Versuchsleiter ${ }^{*}$ in. Die Teilnehmenden wurden über den Sinn und Zweck der Studienteilnahme sowie Datensicherung und Anonymisierung aufgeklärt und erhielten als Aufwandsentscheidung Süßwaren oder eine Vergütung in Form von „credits“ im Rahmen ihres Studiums. Die Teilnehmenden bestätigen ihr Einverständnis zur Studienteilnahme schriftlich. Voraussetzungen für die Studienteilname waren ein Mindestalter von 16 Jahren, keine Farbenblindheit oder Taubheit sowie die regelmäßige Nutzung sozia- 
Auditives Cue-

Reactivity-

Paradigma

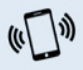

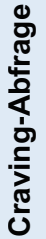

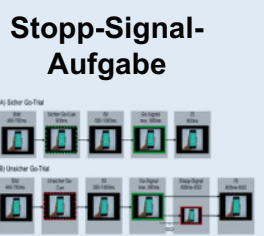

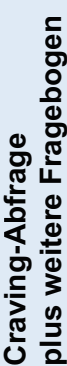

Messzeitpunkt t3
Abb. $1<$ Schematische Darstellung des Studienverlaufs

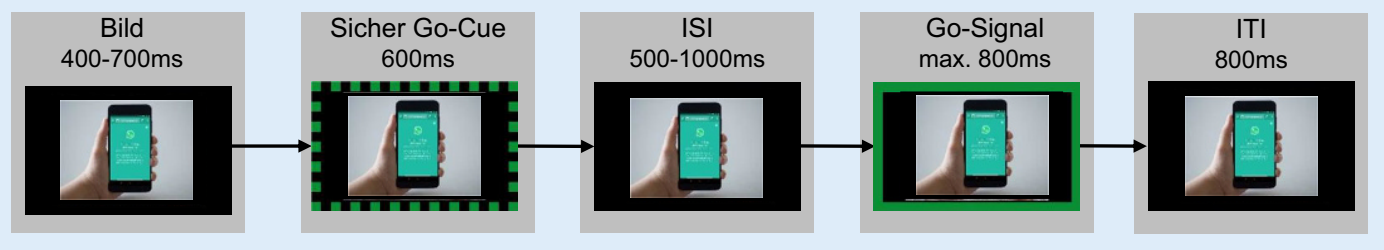

a

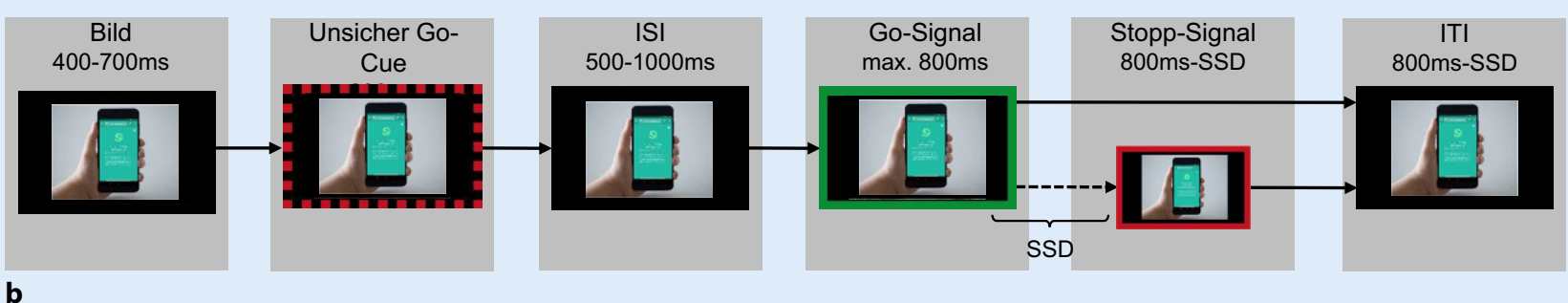

Abb. 2 - Illustration der Stopp-Signal-Aufgabe und Veranschaulichung der sicheren (a) und unsicheren (b) Go-Trials. ISI, inter-stimulus-interval", ITI, inter-trial-interval", SSD "stop-signal delay"

ler Netzwerke. Ein positives Votum der Ethikkommission lag vor. Die Studiendauer umfasste $45 \mathrm{~min}$. Der Verlauf der Studie ist in 0 Abb. 1 dargestellt.

\section{Verwendete Fragebogen}

Die Kurzversion des Internet Addiction Test (Pawlikowski et al. 2013), modifiziert für die Nutzung sozialer Netzwerke und Online-Kommunikationsanwendungen (Wegmann et al. 2015), erfasst die subjektive Symptombelastung im Alltag aufgrund der unkontrollierten Nutzung der spezifischen Anwendungen. Der Fragebogen umfasst 12 Items (z. B. „Wie häufig versuchen Sie, weniger Zeit mit Online-Kommunikationsanwendungen zu verbringen, und schaffen es nicht?"), die auf einer 5-stufigen Skala (1: „nie“" bis 5: „sehr oft“) bewertet und $\mathrm{zu}$ einem Summenwerte von 12 bis maximal 60 addiert werden. Höhere Werte deuten auf eine höhere subjektive Symptombelastung hin.

Zur Erfassung der Impulsivität wurde die deutsche Kurzversion der Barratt Impulsiveness Scale (Meule et al. 2011) eingesetzt. Die 15 Items (z. B. „Ich bin unaufmerksam.") werden auf einer 4-stufigen Skala (0: „nie/selten“ bis 3: „immer/ fast immer") bewertet. In der vorliegenden Studie wurde der Summenwert aller Items verwendet. Höhere Summenwerte deuten auf eine stärker ausgeprägte Impulsivität hin.

Die Erfassung des subjektiv erlebten Cravings erfolgte mithilfe einer modifizierten Version des Desires for Alcohol Questionnaire (Love et al. 1998). Die 14 Items (z. B. „Ich würde nahezu alles tun, um jetzt Online-Kommunikations- anwendungen nutzen zu können.“) werden auf einer 7-stufigen Skala (0: „stimme überhaupt nicht zu“ bis 6: „stimme vollkommen zu") bewertet und zu einem Mittelwert zusammengefasst, wobei höhere Werte ein höher erlebtes Verlangen illustrieren. Der Fragebogen wurde insgesamt an 3 Stellen eingesetzt, um den Verlauf des Cravings während der Studienphase zu erfassen ( $\bullet$ Abb. 1).

\section{Cue-reactivity-Paradigma}

In der vorliegenden Studie wurde ein auditives Cue-reactivity-Paradigma eingesetzt, bei dem die Probanden 12 auditive Reize hinsichtlich ihrer Anregung, Emotionalität und des Verlangens auf einer 5-stufigen Skala (1: ,keine Anregung/ sehr negativ/kein Verlangen“ bis 5: „hohe Anregung/sehr positiv/hohes Verlan- 
3

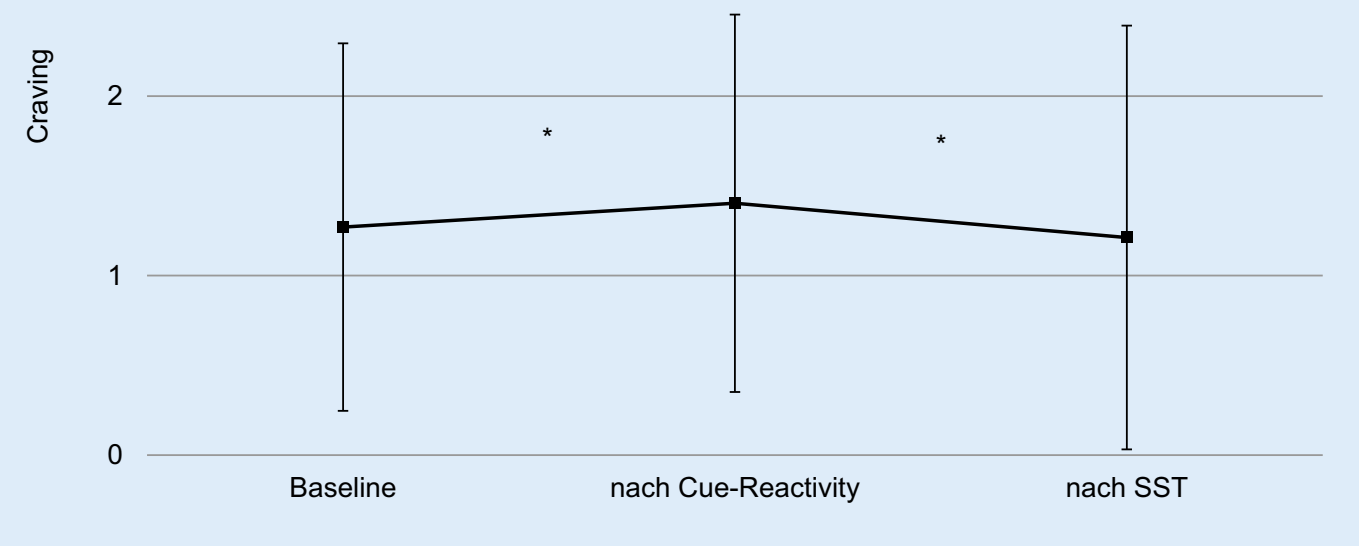

Abb. $3<$ Verlauf des Craving-Erlebens während der Versuchsdurchführung gen“) bewerten sollten. In einer vorherigen Studie konnte bereits gezeigt werden, dass die auditiven Reize als Töne eines Smartphones oder eines sozialen Netzwerks identifiziert werden konnten (z. B. Standardklingelton von WhatsApp; Wegmann et al. 2018). Die Bearbeitungszeit der Aufgabe umfasste ca. $5 \mathrm{~min}$.

\section{„Stop-Signal Task"}

Zur Messung der spezifischen Inhibitionskontrolle wurde eine modifizierte Stopp-Signal-Aufgabe (SST) genutzt, die bereits von Antons und Brand (2018) im Kontext der problematischen Pornografienutzung angewendet wurde (• Abb. 2).

Bei dieser Aufgabe sollen die Teilnehmenden per Tastendruck auf einen grünen Rahmen reagieren. In manchen Durchgängen erscheint kurz nach diesem sog. Go-Signal ein Stopp-Signal (roter Rahmen). Dieser signalisiert, dass die Probanden versuchen sollen, die bereits begonnene Reaktion abzubrechen und, wenn möglich, die Taste nicht zu drücken. Der zeitliche Abstand zwischen dem Go- und dem Stopp-Signal wird Stopp-Signal-Verzögerung („stop-signal delay“, SSD) genannt. Diese wird bei erfolgreicher Inhibition (Taste wurde bei Erscheinen des roten Rahmens nicht gedrückt) um 50 ms erhöht. Schafft der/die
Teilnehmende es nicht, die Reaktion abzubrechen, wird die SSD um $50 \mathrm{~ms}$ verringert. Durch diese Anpassungen wird die Aufgabe an die Inhibitionsleistung des Probanden angepasst und ermöglicht außerdem die Bestimmung der Inhibitionszeit, basierend auf dem "horse-race model“ (Verbruggen et al. 2019). Bevor das Go-Signal erscheint, wird entweder ein sicherer (grün gestrichelter Rahmen) oder unsicherer (rot gestrichelter Rahmen) Go-Cue gezeigt. Diese Hinweisreize informieren die Teilnehmenden darüber, ob in diesem Durchgang ein Stopp-Signal erscheinen kann, oder ob definitiv kein Stopp-Signal erscheinen wird. Während jedes Durchgangs wird stets ein anderes Bild gezeigt, das die Login-Seite eine der 10 in Deutschland meistgenutzten OnlineKommunikationsanwendung zeigt. Dabei handelt es sich um einen distalen Bildreiz, das bedeutet, dass nicht der eigentliche, belohnende Mechanismus (z.B. Unterhaltung in einem Chat oder News-Feed bei Instagram), sondern nur ein damit assoziierter Bildausschnitt (z.B. Anmeldeseite von Instagram) gezeigt wird. Das Bild selbst ist für die Bewältigung der Aufgabe irrelevant, dient jedoch dazu, eine spezifische Inhibitionskontrolle zu messen. Die Aufgabe besteht aus insgesamt 300 Durchgängen
(120 sichere Go-Cues, 180 unsichere GoCues; bei einem Drittel der unsicheren Durchgänge erscheint ein Stopp-Signal) mit einer Dauer von ca. 17 min. Basierend auf Empfehlungen von Verbruggen et al. (2019) wurden die Präsentationszeiten sowie die Zahl der Durchgänge im Vergleich zu der Studie von Antons und Brand (2018; - Abb. 2) angepasst.

\section{Ergebnisse}

Im Rahmen der deskriptiven Analyse des Fragebogens zur Erfassung der Symptomschwere wurde bei 12 Teilnehmenden eine problematische Nutzung (Summenwerte $>30$ ) und bei weiteren 14 Teilnehmenden eine pathologische Nutzung (Summenwerte $>37$ ) der spezifischen Anwendung identifiziert.

Zur Testung der Hypothese 1 wurde der Verlauf des Cravings mithilfe eines Messwiederholungsverfahrens geprüft, um die Konfrontation von spezifischen auditiven Reizen bei dem Erleben von subjektiven Craving zu illustrieren. Die Ergebnisse zeigen, dass infolge des $\mathrm{Pa}$ radigmas das Craving leicht, aber signifikant anstieg. Nach der SST sank das Craving wieder signifikant (• Abb. 3). Für die weiteren Analysen wurde das Craving direkt nach dem Cue-reactivityParadigma verwendet. 
Tab. 1 Deskriptive Kennwerte und bivariate Korrelationen nach Pearson zwischen den Konstrukten der Symptomschwere, Impulsivität, Craving und Inhibitionskontrolle

\section{Variable}

(1) Symptomschwere

(2) Impulsivität

(3) Craving nach "cue reactivity"

(4) Inhibitionszeit

$M$ Mittelwert, SD Standardabweichung

${ }^{*} p \leq 0,05,{ }^{* *} p \leq 0,01$

$\mathbf{M} \pm$ SD

$28,4 \pm 9,1$

$32,4 \pm 6,0$

$1,4 \pm 1,1$

$208,7 \pm 53,7$
(2)

(3)

- - -

$0,317^{*}$

$0,488^{* *}$

0,035

0,025

$-0,178$

$-$

$-$

-

0,159
Zur Testung der Hypothese 2 wurden die Zusammenhänge zwischen der Symptomschwere einer problematischen Nutzung sozialer Netzwerke und den Konstrukten Impulsivität, Craving und spezifischer Inhibitionskontrolle geprüft (- Tab. 1). Es lagen signifikante Zusammenhänge zwischen der Symptomschwere und Impulsivität sowie dem erlebten Craving vor. Kein signifikanter Zusammenhang konnte zwischen der spezifischen Inhibitionskontrolle und der Symptomschwere identifiziert werden.

Als Ergänzung zur zweiten Hypothese wurde eine multiple Regression durchgeführt, die zeigte, dass $33,1 \%$ der Symptomschwerevarianz durch die Variablen Impulsivität, Craving und Inhibitionszeit erklärt werden können. Der Beitrag der Inhibitionszeit war jedoch nicht signifikant. Das Craving wies allerdings auch über die generelle Impulsivität einen signifikanten Prädiktionswert auf (• Tab. 2).

Angelehnt an theoretische Annahmen aus der Suchtforschung und vorherigen empirischen Arbeiten wurden zusätzlich Interaktionseffekte zwischen Impulsivität, Craving und Inhibitionskontrolle angenommen und geprüft (s. Hypothese 3). Moderierte Regressionsanalysen ergaben, dass über die Effekte der einzelnen Komponenten hinaus, die Interaktionen nicht signifikant zur weiteren Varianzaufklärung der Symptomschwere beitrugen (alle $\beta<0,067$, alle $p>0,626$ ).

\section{Diskussion}

Die Ergebnisse der vorliegenden Studie veranschaulichen, dass Impulsivität und das Erleben von Craving relevante Konstrukte bei der problematischen Nutzung sozialer Netzwerke darstellen. Die Konfrontation mit anwendungsspezifischen auditiven Reizen führte zu einem signifikanten Anstieg des Cravings. Die spezifische Inhibitionskontrolle konnte in der vorliegenden Studie keine weitere Varianz der Symptomschwere aufklären; dies galt auch für die Interaktionseffekte zwischen allen 3 untersuchten Konstrukten.

Hinsichtlich der zugrunde liegenden affektiven und kognitiven Komponenten bei der Entwicklung und Aufrechterhaltung einer problematischen Nutzung sozialer Netzwerke wird deutlich, dass Impulsivität einen bedeutsamen Risikofaktor darstellt, was sich mit empirischen Annahmen aus der substanzbezogenen und verhaltensbezogenen Suchtforschung deckt (Brand et al. 2019; Mitchell und Potenza 2014). Ähnlich konnte auch der Zusammenhang zwischen dem erlebten Craving und der Symptomschwere bestätigt werden, wobei auch hier von einem robusten Ergebnis unter Berücksichtigung vorheriger Studien auszugehen ist (Starcke et al. 2018; Wegmann et al. 2018). Auffällig ist jedoch, dass der Craving-Anstieg nach der Konfrontation mit auditiven Reizen zwar signifikant ist, deskriptiv aber relativ gering ausfällt, was sich mit der Studie von Wegmann et al. (2018) deckt. Die spezifische Inhibitionskontrolle sowie Interaktionseffekte konnten anders als in vorherigen Studien (Gao et al. 2019; Wegmann et al. 2020) nicht zur Varianzaufklärung der Symptomschwere beitragen. Grundsätzlich gilt es hier, die unterschiedlich verwendeten Reize genauer zu betrachten. Wegmann et al. (2020) nutzten in dem modifizierten Paradigma auditive Reize mit einem direkten Bezug zu sozialen Netzwerken und dem Smartphone, die möglicherwei-
Tab. 2 Standardisierte Koeffizienten der multiplen Regressionsanalyse zur Varianzaufklärung der Symptomschwere

\begin{tabular}{|c|c|c|c|}
\hline Variable & $\boldsymbol{\beta}$ & $T$ & $p$ \\
\hline Konstante & - & 0,99 & 0,326 \\
\hline Impulsivität & 0,308 & 2,86 & 0,006 \\
\hline $\begin{array}{l}\text { Craving nach "cue } \\
\text { reactivity" }\end{array}$ & 0,478 & 4,46 & $\leq 0,001$ \\
\hline Inhibitionszeit & 0,014 & 0,13 & 0,896 \\
\hline
\end{tabular}

se dadurch einen größeren individuellen Aufforderungscharakter hatten. Die in der vorliegenden Studie genutzten Bildreize, die nicht das mit der Belohnung assoziierte Verhalten direkt (z.B. ChatVerlauf oder Likes bei Instagram), sondern indirekte Hinweisreize (z. B. „login page“ von Instagram auf dem Smartphone) zeigten, könnten möglicherweise $\mathrm{zu}$ generisch für die Aufrechterhaltung des Cravings sein, wobei dies das Craving selbst als auch die Inhibitionsleistung betrifft. Für die Durchführung der StoppSignal-Aufgabe sind darüber hinaus die präsentierten Reize irrelevant. Dadurch haben die Reize im Vergleich zur GoNoGo-Aufgabe kein direktes Konfliktpotenzial und müssen weniger tiefgreifend verarbeitet werden. Dies könnte zu einer verringerten Interaktion zwischen der Verarbeitung der Reize und der Handlungskontrolle geführt haben.

Bezogen auf den Alltag veranschaulicht dies, dass v.a. die auditive oder visuelle Wahrnehmung der genutzten sozialen Netzwerke dazu führt, dass Personen die Aufforderung verspüren, jetzt das Smartphone zu nutzen. Diesem Wunsch nachzugehen, stellt jedoch in den meisten Alltagssituationen (z. B. im öffentlichen Nahverkehr, bei der Arbeit) keine real wahrgenommene Konfliktsituation dar, sondern möglicherweise nur eine erste Ablenkung. Das Prüfen der sozialen Netzwerke oder einer Nachricht erfolgt einfach und unkompliziert, was gleichzeitig eine geringere Deprivation und auch eine geringere Handlungskontrolle bezüglich des Erlebens von Craving oder von einer Konfliktsituation bei den Nutzerinnen und Nutzern benötigt. Bei der Betrachtung einer problematischen Nutzung sozialer Netzwerke im Vergleich $\mathrm{zu}$ anderen suchtartigen Verhaltenswei- 
sen beschreibt dies möglicherweise ein zentrales Kriterium, wobei damit einhergehend negative Konsequenzen und subjektive Beeinträchtigungen ebenfalls geringer ausfallen könnten.

Bei der Einordnung der vorliegenden Ergebnisse gilt es jedoch, mögliche Limitationen zu berücksichtigen. Im Hinblick auf die Stichprobe muss einschränkend konstatiert werden, dass eine subjektive Selbsteinschätzung des Störungsbildes und keine klinische Diagnostik vorgenommen wurde. Dabei betrug der Anteil derjenigen, die bereits von ersten Einschränkungen im Alltag aufgrund einer unkontrollierten Nutzung sozialer Netzwerke berichteten, insgesamt ca. $40 \%$ und war damit erstaunlich hoch. Dies könnte natürlich auch die Bearbeitung der einzelnen Aufgaben und v. a. die Verarbeitung der spezifischen Reize maßgeblich beeinflusst haben. Im Hinblick auf die Stichprobengröße gilt es, diese Effekte in weiteren Studien zu adressieren, aber gleichzeitig erscheint - um eine Überschätzung des Störungsbildes zu vermeiden - der Einsatz standardisierter, diagnostischer Verfahren zur verbesserten Einschätzung der Prävalenzen zwingend notwendig. Auch die verwendete Stopp-Signal-Aufgabe mitsamt der Bildreize müsste in zukünftigen Studien hinsichtlich der Validität geprüft werden. Hier wäre außerdem eine vergleichende Betrachtung auditiver und visueller Reize innerhalb des Störungsbildes als auch im Vergleich zu weiteren Verhaltenssüchten wünschenswert.

\section{Schlussfolgerung und Ausblick}

Betrachtet man die vorliegenden Ergebnisse vor dem Hintergrund der Diskussion, ob es sich bei dem Störungsbild tatsächlich um ein suchtartiges Verhalten handelt, bietet es sich an, die zuvor genannten 3 Kriterien von Brand et al. (2020) hinzuzuziehen. Es kann zwar keine abschließende Aussage hinsichtlich der klinischen Relevanz getroffen werden, doch die Ergebnisse lassen zumindest eine erste Einschätzung der Problematik zu. Darüber hinaus erlauben die Hinzunahme theoretischer Annahmen und die Prüfung zugrunde liegender Mechanismen die Schlussfolgerung, dass ein
Vergleich mit anderen Störungsbildern wie dem pathologischen Glücksspielen und Videospielen möglich ist. Es scheint jedoch genauso wichtig, die Abgrenzung zu den genannten Störungsbildern als auch mögliche Alleinstellungsmerkmale zu identifizieren. Dies betrifft sowohl die Spezifität einzelner zugrunde liegender Mechanismen als auch theoretischer Konzepte, um neben der Bestimmung konvergenter Validitäten auch die divergenten Prozesse eines Störungsbildes zu verstehen.

Der Ausblick für zukünftige Studien sollte somit darin bestehen, weiterhin zugrunde liegende Mechanismen zwischen den einzelnen problematischen Verhaltensweisen $\mathrm{zu}$ untersuchen und zu identifizieren. Darüber hinaus ist die enge Verzahnung zwischen der Nutzung sozialer Netzwerke und dem Smartphone ein wichtiger Faktor im täglichen Gebrauch, der neben klinischen Studienansätzen auch die Notwendigkeit der methodischen Vielfalt unterstreicht. So könnte beispielsweise der Einsatz eines „ambulatory assessment“ zur direkten Abfrage von erlebten Konflikten in der Handlungskontrolle, aber auch dem Erleben von Craving, Deprivation sowie Bedürfnisbefriedigung einen vielversprechenden Ansatz darstellen.

\section{Fazit für die Praxis}

\section{- Impulsivität und das Erleben von Craving scheinen relevante Merkmale einer problematischen Nutzung sozialer Netzwerke zu sein. \\ - Beeinträchtigungen in der Inhi- bitionskontrolle scheinen nicht notwendigerweise mit einer proble- matischen Nutzung einherzugehen. \\ - Die Spezifität und selbstverständli- che Integration der Nutzung im Alltag sollten in weiterführenden Studien gezielt adressiert werden.}

\section{Korrespondenzadresse}

\section{Dr. Elisa Wegmann}

Allgemeine Psychologie: Kognition und Center for Behavioral Addiction Research (CeBAR), Universität Duisburg-Essen

Forsthausweg 2, 47057 Duisburg, Deutschland elisa.wegmann@uni-due.de
Funding. Open Access funding enabled and organized by Projekt DEAL.

\section{Einhaltung ethischer Richtlinien}

Interessenkonflikt. E. Wegmann, K.-S. Jung und $S$. Antons geben an, dass kein Interessenkonflikt besteht.

Für diesen Beitrag wurde eine Studie mit Probanden durchgeführt. Für diese Studie liegt ein Ethikantrag der zuständigen Ethikkommission der Abteilung für Informatik und Angewandte Kognitionswissenschaft der Fakultät für Ingenieurwissenschaften der Universität Duisburg-Essen vor.

Open Access. Dieser Artikel wird unter der Creative Commons Namensnennung 4.0 International Lizenz veröffentlicht, welche die Nutzung, Vervielfältigung, Bearbeitung, Verbreitung und Wiedergabe in jeglichem Medium und Format erlaubt, sofern Sie den/die ursprünglichen Autor(en) und die Quelle ordnungsgemäß nennen, einen Link zur Creative Commons Lizenz beifügen und angeben, ob Änderungen vorgenommen wurden.

Die in diesem Artikel enthaltenen Bilder und sonstiges Drittmaterial unterliegen ebenfalls der genannten Creative Commons Lizenz, sofern sich aus der Abbildungslegende nichts anderes ergibt. Sofern das betreffende Material nicht unter der genannten Creative Commons Lizenz steht und die betreffende Handlung nicht nach gesetzlichen Vorschriften erlaubt ist, ist für die oben aufgeführten Weiterverwendungen des Materials die Einwilligung des jeweiligen Rechteinhabers einzuholen.

Weitere Details zur Lizenz entnehmen Sie bitte der Lizenzinformation auf http://creativecommons.org/ licenses/by/4.0/deed.de.

\section{Literatur}

American Psychiatric Association (2013) Diagnostic and statistical manual of mental disorders, 5. Aufl. American Psychiatric Publishing, Washington DC Andreassen CS (2015) Online social network site addiction: a comprehensive review. Curr Addict Rep 2:175-184. https://doi.org/10.1007/s40429015-0056-9

Antons S, Brand M (2018) Trait and state impulsivity in males with tendency towards Internet-pornography-use disorder. Addict Behav 79:171-177. https://doi.org/10.1016/j.addbeh.2017.12.029

Bari A, Robbins TW (2013) Inhibition and impulsivity: behavioral and neural basis of response control. Progess Neurobiol 108:44-79. https://doi.org/ 10.1016/j.pneurobio.2013.06.005

Bechara A (2005) Decision making, impulse control and loss of willpower to resist drugs: a neurocognitive perspective. Nat Neurosci 8:1458-1463

Boyd DM, Ellison NB (2007) Social network sites: definition, history, and scholarship. J Comput Commun 13:210-230. https://doi.org/10.1111/ j.1083-6101.2007.00393.x

Brand M, Rumpf H-J, Demetrovics Z, Müller A, Stark R, King DL, Potenza MN et al (2020) Which conditions should be considered as disorders in the International Classification of Diseases (ICD11) designation of "other specified disorders due 
to addictive behaviors"? J Behav Addict. https:// doi.org/10.1556/2006.2020.00035

Brand M, Wegmann E, Stark R, Müller A, Wölfling K, Robbins TW, Potenza MN (2019) The Interaction of Person-Affect-Cognition-Execution (I-PACE) model for addictive behaviors: update, generalization to addictive behaviors beyond Internetuse disorders, and specification of the process character of addictive behaviors. Neurosci Biobehav Rev 104:1-10. https://doi.org/10.1016/j. neubiorev.2019.06.032

Carter BL, Tiffany ST (1999) Meta-analysis of cuereactivity in addiction research. Addiction 94:327-340

Clayton RB, Osborne RE, Miller BK, Oberle CD (2013) Loneliness, anxiousness, and substance use as predictors of Facebook use. Comput Human Behav 29:687-693. https://doi.org/10.1016/j. chb.2012.12.002

DAK-Gesundheit (2018) WhatsApp, Instagram und Co. - so süchtig macht Social Media (DAK-Studie: Befragung von Kindern und Jugendlichen zwischen 12 und 17 Jahren). https://www.dak. de/dak/download/dak-studie-social-medianutzung-1968596.pdf. Zugegriffen: 4. März 2018

Gao Q, Jia G, Zhao J, Zhang D (2019) Inhibitory control in excessive social networking users: evidence from an event-related potential-based Go-Nogo task. Front Psychol 10:1810. https://doi.org/10. 3389/fpsyg.2019.01810

Guedes E, Sancassiani F, Carta MG, Campos C, Machado S, King ALS, Nardi AE (2016) Internet addiction and excessive social networks use: what about Facebook? Clin Pract Epidemiol Ment Health 12:43-48. https://doi.org/10.2174/ 1745017901612010043

Kim YJ, Lim JA, Lee JY, Oh S, Kim SN, Kim DJ, Choi JS et al (2017) Impulsivity and compulsivity in Internet gaming disorder: a comparison with obsessivecompulsive disorder and alcohol use disorder. J Behav Addict 6:545-553. https://doi.org/10. 1556/2006.6.2017.069

Kuss DJ, Griffiths MD (2011) Online social networking and addiction: a review of the psychological literature. Int J Enviromental Res Public Health 8:3528-3552. https://doi.org/10.3390/ ijerph8093528

Love A, James D, Willner P (1998) A comparison of two alcohol craving questionnaires. Addiction 93:1091-1102

Meule A, Vögele C, Kübler A (2011) Psychometrische Evaluation der deutschen Barratt Impulsiveness Scale - Kurzversion (BIS-15). Diagnostica 57:126-133. https://doi.org/10.1026/0012 1924/a000042

Mitchell MR, Potenza MN (2014) Addictions and personality traits: Impulsivity and related constructs. Curr Behav Neurosci Rep 1:1-12. https://doi.org/10.1007/s40473-013-0001-y

Montag C, Wegmann E, Sariyska R, Demetrovics Z, Brand M (2019) How to overcome taxonomical problems in the study of Internet use disorders and what to do with "smartphone addiction"? JBA. https://doi.org/10.1556/2006.8.2019.59

Moreau A, Laconi S, Delfour M, Chabrol H (2015) Psychopathological profiles of adolescent and young adult problematic Facebook users. Comput Human Behav 44:64-69. https://doi. org/10.1016/j.chb.2014.11.045

Pawlikowski M, Altstötter-Gleich C, Brand M (2013) Validation and psychometric properties of a short version of Young's Internet Addiction Test.
Comput Human Behav 29:1212-1223. https:// doi.org/10.1016/j.chb.2012.10.014

Robinson TE, Berridge KC (2008) The incentive sensitization theory of addiction: some current issues. Philos Trans Royal Soc B Biol Sci 363:3137-3146. https://doi.org/10.1098/rstb. 2008.0093

Starcke K, Antons S, Trotzke P, Brand M (2018) Cuereactivity in behavioral addictions: a metaanalysis and methodological considerations. J Behav Addict 7:227-238. https://doi.org/10. 1556/2006.7.2018.39

Verbruggen F, Aron AR, Band GP, Beste C, Bissett PG, Brockett AT, Boehler CN et al (2019) A consensus guide to capturing the ability to inhibit actions and impulsive behaviors in the stop-signal task. Elife. https://doi.org/10.7554/eLife.46323

Wegmann E, Brand M (2019) A narrative overview about psychosocial characteristics as risk factors of a problematic social networks use. Curr Addict Rep 6:402-409. https://doi.org/10.1007/ s40429-019-00286-8

Wegmann E, Brand M (2020) Cognitive correlates in gaming disorder and social networks use disorder: a comparison. Curr Addict Rep 7:356-364. https://doi.org/10.1007/s40429020-00314-y

Wegmann E, Stodt B, Brand M (2015) Addictive use of social networking sites can be explained by the interaction of Internet use expectancies, Internet literacy, and psychopathological symptoms. J Behav Addict 4:155-162. https://doi.org/10. 1556/2006.4.2015.021

Wegmann E, Stodt B, Brand M (2018) Cue-induced craving in Internet-communication disorder using visual and auditory cues in a cue-reactivity paradigm. Addict Res Theory 26:306-314. https:// doi.org/10.1080/16066359.2017.1367385

Wegmann E, Müller SM, Turel O, Brand M (2020) Impulsivity, general executive functions, and specific inhibitory control in social-networksuse disorder. Sci Rep 10:3866. https://doi.org/10. 1038/s41598-020-60819-4

World Health Organization (2020) Website for ICD-11 for Mortality and Morbidity Statistics. https:// icd.who.int/browse11/l-m/en\#/http://id.who. int/icd/entity/1448597234. Zugegriffen: 13. Jan. 2021

\section{Dem digitalen Placebo- Effekt auf der Spur}

In einer randomisierten-kontrollierten

Studie, die Forschende der Internationalen Psychoanalytischen Universität (IPU) Berlin, gemeinsam mit Forschenden der Universität Basel und der RWTH Aachen veröffentlicht haben, konnte ein digitaler Placebo-Effekt gezeigt werden. Die Ergebnisse bilden eine wichtige Grundlage, um Placebo-Effekte im Rahmen digitaler Gesundheitsanwendungen (DiGA) individuell zu nutzen.

In der Studie untersuchten Prof. Dr. Gunther Meinlschmidt und KollegInnen der IPU Berlin, der Universität Basel sowie der RWTH Aachen die Veränderung der Wirkungserwartung einer für die Untersuchung programmierten Smartphone-App über einen Zeitraum von drei Wochen. Die 132 gesunden Probandlnnen wurden in vier Gruppen aufgeteilt. Der ersten Gruppe wurde vorab mitgeteilt, dass eine Wirkung zu erwarten sei, die zweite bekam erst im Nachhinein an einzelnen Tagen mitgeteilt, dass eine Wirkung der App tatsächlich eingetreten sei. In der dritten Gruppe wurde beides miteinander kombiniert, die vierte Gruppe erhielt keine der beiden Mitteilungen.

Die Ergebnisse zeigen: »Werden Informationen zur Wirksamkeit vor und nach Nutzung von Smartphone-Apps kombiniert, könnten dadurch Wirkungserwartung und auch Glaubwürdigkeit digitaler Gesundheitsanwendungen nachhaltiger werden«, so Meinlschmidt. Das könne beispielsweise helfen, weniger PatientInnen zu verlieren, was eine große Herausforderung bei DiGA darstelle.

Originalpublikation: Stalujanis $\mathrm{E}_{\text {, }}$ Neufeld J, Glaus Stalder M, Belardi A, Tegethoff M, Meinlschmidt G Induction of Efficacy Expectancies in an Ambulatory Smartphone-Based Digital Placebo Mental Health Intervention: Randomized Controlled Trial JMIR Mhealth Uhealth 2021;9(2):e20329 doi: $10.2196 / 20329$

Quelle: International Psychoanalytic University Berlin (www.ipu-berlin.de [17.02.2021]) 